\title{
Bunching for Shorter Damping Rings for the ILC
}

\author{
David Neuffer \\ Fermilab, PO Box 500, Batavia IL 60510
}

\begin{abstract}
A variant rearrangement of the bunch trains for the ILC that enables much shorter damping rings is presented. In a particular example the $\sim 2280$ bunches are regrouped into $\sim 450$ subtrains of five adjacent bunches. These subtrains are extracted from the damping rings at $\sim 2.2 \mu$ s intervals, obtaining the $1 \mathrm{~ms}$ macrobunch length of the baseline TESLA collider scenario. If the baseline damping rf frequency is $325 \mathrm{MHz}$ and the kicker rise and fall times are $\sim 20 \mathrm{~ns}$, a ring circumference of $\sim 4.5 \mathrm{~km}$ is required. Variations of the scheme could easily reduce the circumference to $\sim 3 \mathrm{~km}$, and faster kickers could reduce it even further.
\end{abstract}

\section{Introduction}

In the baseline TESLA design, streams of $\sim 2280$ electron and positron bunches are collided, with the bunches evenly spaced over a $1 \mathrm{~ms}$ spill time (300000m). [1]

A key difficulty in the TESLA design is the unwieldy length of the damping rings ( $\sim 17 \mathrm{~km}$ each). This length greatly increases cost, and gives the beams undesirably large space charge effects. This circumference was set by requiring that the ring store 2280 bunches and that the bunches be separated by the rise and fall time of the injection/extraction kickers, which are set at $\sim 20 \mathrm{~ns}$. Schemes that reduce the required circumference of the rings by reducing the kicker rise and/or fall times are being developed. In the present note we consider allowing the kicker to extract a finite number of bunches, separated by the damping rf wavelength.

To simplify the discussion we require that the damping ring rf frequency be a subharmonic of the main Linac rf frequency, which is $1300 \mathrm{MHz}$ with TESLA cavities. In the present case this means $1300,650,433$, or $325 \mathrm{MHz}$ rf. With this constraint every rf bucket in the damping ring is properly timed to provide an accelerated bunch in the linac, and the maximum possible flexibility in bunch scenarios is maintained. (The 2002 TESLA design had $500 \mathrm{MHz}$ rf, and the buckets match the linac cycle only in one of 5 rf cycles; The 1998 design had $433 \mathrm{MHz}$; an integer 3 subharmonic.)

With $325 \mathrm{MHz}$ rf, 2280 bunches requires a circumference of at least $2104 \mathrm{~m} ; 650 \mathrm{MHz}$ requires $1057 \mathrm{~m}$ (with no extra gaps for injection/extraction etc.). A 20ns bunch to bunch spacing implies a minimum circumference of $13.7 \mathrm{~km}$; requiring a complete extraction kick spacing between each bunch sets the scale of the damping ring circumferences. We propose to reduce the ring circumference by grouping the bunches into subtrains separated by the extraction gap. 


\section{Examples}

To generate some numerical examples, we choose a $325 \mathrm{MHz}$ damping ring rf system, set the number of bunches per subtrain to $5\left(\mathrm{n}_{\mathrm{bt}}=5\right)$. We set the extraction kicker rise and fall times to $20 \mathrm{~ns}(6 \mathrm{~m})$, which corresponds to $\sim 6.5$ wavelengths. We set a 7 wavelength spacing between bunch trains, making the gap + bunch subtrain length equal to 11 wavelengths or $10.154 \mathrm{~m}$. (An extraction pulse and bunch subtrain is shown in fig. 1.) To obtain 2280 total bunches we need 456 such units or $\sim 4.63 \mathrm{~km}$ ring circumference. This is $\sim 1 / 4$ of the Tesla damping ring.

To determine a damping ring circumference with evenly spaced extractions, we can set up a bunch configuration such that the extraction kickers fire at constant intervals during the $1 \mathrm{~ms}$ spill, with the interval matched to an appropriate integer multiple of the $10.154 \mathrm{~m}$ bunch + gap subtrain unit length. A simple division of 300000 by 456 obtains $658 \mathrm{~m}$, closest to 65 subtrains. If the spacing between kicks is 65 subtrains and the circumference of the ring is $(65 \times \mathrm{N} \pm 1)$ subtrains long, where $\mathrm{N}$ is any integer, then ring will empty with evenly spaced kicks. We choose $\mathrm{N}$ to be the closest to $65 \times \mathrm{N}=456$ or $\mathrm{N}=7$, obtaining a final circumference of $(65 \times 7+1) \times 10.154=$ $456 \times 10.154=4630 \mathrm{~m}$. (Note that slightly unevenly spaced kicks to change the loading/unloading scenario are probably not very difficult.)

We can shift the numerology by choosing a $650 \mathrm{MHz}$ rf system $(\lambda=0.4615 \mathrm{~m})$, and retain 5 bunches per train. The reference time of $20 \mathrm{~ns}$ is close to 14 wavelengths and our gap + bunch subtrain length becomes 18 wavelengths or $8.307 \mathrm{~m}$. With 456 such units we need a circumference of $3788 \mathrm{~m}$, almost a $\mathrm{km}$ less than the $325 \mathrm{MHz}$ example.

The numerology for even bunch trains is a bit less matched than the previous case (which came out exactly). $658 \mathrm{~m}$ corresponds to 79 complete subtrains and we can choose $\mathrm{N}=6$ (with 79) obtaining a circumference of $(6 \times 79-1) 8.307=3946 \mathrm{~m}$. (This numerical exercise gives us a slightly larger total number of bunches, but the numbers could be reshifted to obtain a smaller number. For example, reducing the baseline spacing to 76 , with $\mathrm{N}=6$, obtains $456 \pm 1$ subtrains and $3788 \pm 8 \mathrm{~m}$ circumference.)

The numbers in the examples cited above depend on the values of the number of bunches per subtrain, the rf wavelength, the extraction gap length, spill length, and all of these numbers can change when the operational choices are changed, but the overall scenario can still be reconstructed and readapted to the parameter variations. Note that the parameters of the ring should be chosen to maintain maximum flexibility in redefining the bunch spacing and arrangements, allowing for improvements in kicker performance, and maintaining the possibility of increasing luminosity by filling more buckets. Requiring the $\mathrm{rf}$ to be an exact linac subharmonic maintains that type of flexibility.

This particular example used 5 bunches per subtrain; similar scenarios with $2,3,4,5,6, \ldots$ bunches could be developed, with similar properties. Within a scenario, the number of bunches per subtrain could be varied operationally to increase luminosity or reduce beamstrahlung, provided the extraction kick is accommodated. ... (One bunch per subtrain recovers the TDR scenario.) More than $\sim 6$ would probably be too unwieldy. 


\section{Potential difficulties}

The bunch reconfiguration does change some of the Linear Collider operating conditions. The bunch trains will deplete the rf cavities a bit unevenly, with a bit more field at the head than the tail, and some correction may be needed. The correction required should be small; much smaller than that used in NLC scenarios. To estimate the potential effect, we note that each ILC bunch has $2 \times 10^{10}$ electrons (or positrons), and acceleration through a $\sim 1 \mathrm{~m}$ long TESLA 9-cell cavity requires $\sim 0.096 \mathrm{~J}$. The total energy stored in the TESLA cavity is given by:

$$
\mathrm{U}=\frac{\mathrm{V}_{\mathrm{t}}{ }^{2}}{\mathrm{R} / \mathrm{Q} \omega_{0}}
$$

The factor R/Q is $\sim 1000 \Omega$ for the TESLA $1300 \mathrm{MHz}$ cavities, and $\mathrm{V}_{\mathrm{t}}$ is $\sim 30 \mathrm{MV}$. The resulting value of $\mathrm{U}$ is $\sim 110 \mathrm{~J}$. Thus the bunch only removes $\sim 10^{-3}$ of the cavity energy, which implies a gradient drop of $\sim 0.5 \times 10^{-3}$ if uncompensated, increasing to $\sim 2 \times 10^{-3}$ with 4 leading bunches. This could lead to a corresponding energy variation in the final beam. While compensation will not be absolutely necessary, it would be desirable, and an optimal scheme is left as an exercise to the reader.

Some care must be taken to insure that the different bunches do not overlap in the collision area (crossing at small angle or ...). Note that the NLC design has bunches spaced at $714 \mathrm{MHz}(1.4$ $\mathrm{ns})$, more closely than even the $650 \mathrm{MHz}$ spacing.

The kicker pulse needs a rise time of 20ns, a flat top sufficient to deflect all of the subtrain bunches ( $\sim 12.5 \mathrm{~ns}$ for $325 \mathrm{MHz}, \sim 6.5 \mathrm{~ns}$ for $650 \mathrm{MHz}$ with a 5-bunch flattop), and a 20ns fall time, as schematically shown in fig. 1 . This should not be too difficult.

We have not designed the injector into the damping ring, except that it must provide bunches matched to the desired pattern. A predamper/buncher for the positrons may (as always) be desirable.

\section{Summary}

A shorter ILC damping ring that maintains the same total number of beam bunches in collision as the Tesla design Report, with the same extraction kicker rise and fall times, can be obtained by rearranging the bunches into small trains. Some potential schemes are presented and others may be developed by simple variations. To maintain maximum flexibility, the damping ring $\mathrm{rf}$ frequency should be an exact rf subharmonic of the Linac rf frequency. (This is true for any damping ring scenario. For example, in the TESLA damping ring scenario the luminosity could be doubled simply by filling two adjacent bunches, after obtaining a slightly faster kicker.)

\section{References}

[1] TESLA Technical Design Report, http://tesla.desy.de/new_pages/TDR_CD/start.html (March, 2001). 


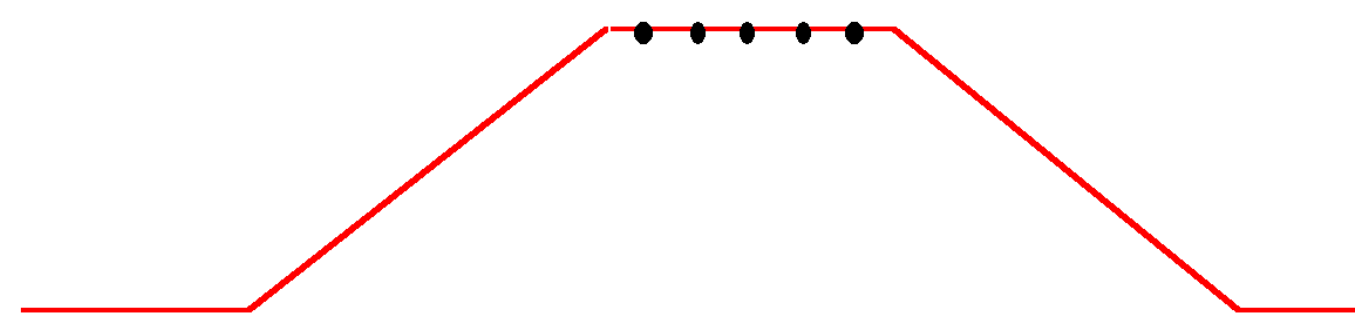

Fig. 1. Schematic view of an extraction pulse with an extended flattop suitable for extracting 5 evenly spaced bunches. Rise and fall times are roughly equal and the flattop is a bit more than half the rise time. 